TRANSACTIONS OF THE

AMERICAN MATHEMATICAL SOCIETY

Volume 302, Number 2, August 1987

\title{
$K$-THEORY AND RIGHT IDEAL CLASS GROUPS FOR HNP RINGS
}

\author{
TIMOTHY J. HODGES
}

\begin{abstract}
Let $R$ be an hereditary Noetherian prime ring, let $S$ be a "Dedekind closure" of $R$ and let $T$ be the category of finitely generated $S$-torsion $R$-modules. It is shown that for all $i \geq 0$, there is an exact sequence $0 \rightarrow$ $K_{i}(\tau) \rightarrow K_{i}(R) \rightarrow K_{i}(S) \rightarrow 0$. If $i=0$, or $R$ has finitely many idempotent ideals then this sequence splits.

A notion of "right ideal class group" is then introduced for hereditary Noetherian prime rings which generalizes the standard definition of class group for hereditary orders over Dedekind domains. It is shown that there is a decomposition $K_{0}(R) \cong \mathrm{Cl}(R) \oplus F$ where $F$ is a free abelian group whose rank depends on the number of idempotent maximal ideals of $R$. Moreover there is a natural isomorphism $\mathrm{Cl}(R) \cong \mathrm{Cl}(S)$ and this decomposition corresponds closely to the splitting of the above exact sequence for $K_{0}$.
\end{abstract}

1. Introduction. This paper is primarily concerned with describing the $K$ groups $K_{i}(R)$ (as defined by Quillen in [13]) for $R$ an hereditary Noetherian prime ring (abbreviated to HNP ring). Two fundamentally different approaches are taken. First of all, by taking a purely categorical point of view, we obtain an expression for $K_{i}(R)$ in terms of $K_{i}(S)$ where $S$ is a sort of "locally finite" torsion-theoretic localization of $R$, and $K_{i}(\tau)$ where $\tau$ is the corresponding torsion class. When $R$ has finitely many idempotent maximal ideals, the most interesting case occurs when $S$ is a Dedekind prime ring right equivalent to $R$. For a general HNP ring, we define a set of "Dedekind closures" of $R$ which coincide in the above case to the Dedekind prime rings right equivalent to $R$. Again the theory applies to such a Dedekind closure $S$, yielding short exact sequences $0 \rightarrow K_{i}(\tau) \rightarrow K_{i}(R) \rightarrow K_{i}(S) \rightarrow 0$ for all $i \geq 0$. Furthermore $K_{i}(\tau)$ may be identified as the direct sum of $K_{i}(R / M)$ where $M$ ranges over some restricted set of idempotent maximal ideals.

The second approach is a more detailed analysis of $K_{0}(R)$ using the fine structure of HNP rings worked out by Goodearl and Warfield in $[6,7]$. We define a notion of "right ideal class group" $\mathrm{Cl}(R)$ generalizing the classical definition for Dedekind domains and the definition for hereditary orders over Dedekind domains in $[\mathbf{1 4}$, p. 343]. We show that $K_{0}(R) \cong \mathrm{Cl}(R) \oplus H_{0}(R)$ where $H_{0}(R)$ is a free group whose rank depends on the number of maximal idempotent ideals of $R$. It turns out that this class group is invariant under Dedekind closure (generalizing a result of Jacobinski for hereditary orders [14, Theorem 40.16]). Thus by splitting off a

Received by the editors July 22, 1985 and, in revised form, August 12, 1986.

1980 Mathematics Subject Classification (1985 Revision). Primary 16A14, 16A54; Secondary $18 \mathrm{~F} 25$.

Key words and phrases. Hereditary Noetherian prime ring, $K$-theory, class group.

(C) 1987 American Mathematical Society $0002-9947 / 87 \$ 1.00+\$ .25$ per page 
suitable rank one summand from $H_{0}(R)$, there are natural isomorphisms $\mathrm{Cl}(R) \oplus$ $Z \cong K_{0}(S)$ and $\bar{H}_{0}(R) \cong K_{0}(\tau)$. Thus the splitting described above corresponds very closely to the exact sequence obtained in the first section.

2. Exactness of $K_{i}(-)$. In this section we prove some general results about when Quillen's long exact localization sequence of $K$-groups [13] splits into short exact sequences. We will be interested in generalized torsion-theoretic localization and we here recall some of the concepts described in [19]. A perfect right localization of a ring $R$ is an epimorphism (in the category of rings) $\phi: R \rightarrow S$ such that $S$ becomes a flat left $R$-module. The crucial property of such extensions is that $S \otimes_{R} S \cong S$ as $S$-S bimodules. This implies that if $\phi_{*}:$ Mod- $R \rightarrow \operatorname{Mod}-S$ is the functor $M \rightarrow M \otimes_{R} S$ and $\phi^{*}: \operatorname{Mod}-S \rightarrow \operatorname{Mod}-R$ is the restriction functor then $\phi_{*} \phi^{*}$ is naturally equivalent to the identity functor on Mod- $S[\mathbf{1 9}, \mathrm{XI} .1 .2]$.

Our $K$-theoretic notation will mostly follow [13]. If $R$ is a right Noetherian ring we denote the category of finitely generated right $R$-modules by $\operatorname{Mod} f-R$. We let $K_{i}^{\prime}(R)=K_{i}(\operatorname{Mod} f-R)$ and $K_{i}(R)=K_{i}(P(R))$ where $P(R)$ is the category of finitely generated projective right $R$-modules. Our main tool will be the localization theorem [13, §5, Theorem 5]. Let $S$ be a perfect right localization of $R$ and let $\tau$ be the subcategory of Mod $f$ - $R$ consisting of $S$-torsion modules, that is modules $M$ such that $M \otimes_{R} S=0$. Then $\tau$ is a Serre subcategory of Mod $f$-R such that the quotient category is Mod $f$-S. Hence we have a long exact sequence of $K$-groups in this situation. We first observe that if $S$ is also finitely generated as an $R$-module, then this long exact sequence splits into short exact sequences.

At various points, our categories of modules need to be small categories (in the sense that the objects form a set) in order for results from [13] to apply. However we can always replace these categories with an equivalent small category by taking a single module from each isomorphism class. We make no further mention of this fact and we shall assume implicitly that we have passed to the small equivalent category whenever necessary.

For readers unfamiliar with higher $K$-theory, it should be noted that the following results can be obtained for $K_{0}$ using only the definitions and results in [1].

THEOREM 2.1. Let $R$ and $S$ be right Noetherian rings and let $\phi: R \rightarrow S$ be a perfect right localization. Let $\tau$ be the torsion subcategory of $\operatorname{Mod} f-R$ consisting of modules $M$ such that $M \otimes_{R} S=0$. If $S$ is finitely generated as a right $R$-module, then the long exact localization sequence splits into split short exact sequences,

$$
0 \rightarrow K_{i}(\tau) \rightarrow K_{i}^{\prime}(R) \rightarrow K_{i}^{\prime}(S) \rightarrow 0 .
$$

ProOF. Applying $K_{*}(-)$ to the short exact sequence of Noetherian categories,

$$
\tau \hookrightarrow \operatorname{Mod} f-R \rightarrow \operatorname{Mod} f-S
$$

yields, by $[\mathbf{1 3}, \S 5$, Theorem 5$]$ the long exact sequence,

$$
\cdots \rightarrow K_{i+1}^{\prime}(S) \stackrel{\partial}{\rightarrow} K_{i}(\tau) \rightarrow K_{i}^{\prime}(R) \stackrel{K_{i}\left(\phi_{*}\right)}{\longrightarrow} K_{i}^{\prime}(S) \stackrel{\partial}{\rightarrow} K_{i-1}(\tau) \rightarrow \cdots
$$

where $\phi_{*}: \operatorname{Mod} f-R \rightarrow \operatorname{Mod} f-S$ is given by $-\otimes_{R} S$. If $\phi^{*}: \operatorname{Mod} f-S \rightarrow \operatorname{Mod} f-R$ is the restriction functor, then as noted above, $\phi_{*} \phi^{*}$ is equivalent to the identity functor whence $K_{i}\left(\phi_{*}\right) K_{i}\left(\phi^{*}\right)$ is the identity on $K_{i}^{\prime}(S)$. Hence the connecting maps $\partial$ are zero and the sequence splits into split short exact sequences

$$
0 \rightarrow K_{i}(\tau) \rightarrow K_{i}^{\prime}(R) \rightarrow K_{i}^{\prime}(S) \rightarrow 0
$$


COROLlaRY 2.2. Let $R, S$ and $\tau$ be as above. If all finitely generated right $R$-modules have finite projective dimension, then

$$
K_{i}(R) \cong K_{i}(S) \oplus K_{i}(\tau) .
$$

EXAMPLE. As an example we analyze the $K$-theory of $R=\left(\begin{array}{cc}\mathrm{Z} & \mathrm{Z} \\ n \mathrm{Z} & \mathrm{Z}\end{array}\right)$. Let $S$ be the $2 \times 2$ matrix $\operatorname{ring}$ over $Z$. Since $S$ and $R$ contain a common right (resp. left) ideal $I$ such that $S I=S$ (resp. $I S=S$ ), it follows from $[\mathbf{1 5}, 2.1]$ that $S$ is finitely generated and projective as both a left and a right $R$-module. Hence $S$ is a perfect right localization of $R$. It is not difficult to see that the subcategory of finitely generated $S$-torsion $R$-modules is naturally equivalent to $\operatorname{Mod} f-(Z / n Z)$. Hence if $n=p_{1}^{r_{1}} \cdots p_{t}^{r_{t}}$ for some primes $p_{1}, \ldots, p_{t}$ and positive integers $r_{1}, \ldots, r_{t}$, then

$$
K_{i}(\tau) \cong \bigoplus_{j} K_{i}^{\prime}\left(Z / p_{j}^{r_{j}} Z\right) \cong \bigoplus_{j} K_{i}\left(Z / p_{j} Z\right)
$$

by devissage $[13, \S 5$, Theorem 4]. Thus, by Theorem 2.1 ,

$$
K_{i}^{\prime}(R) \cong K_{i}(Z) \oplus\left(\bigoplus_{j} K_{i}\left(Z / p_{j} Z\right)\right)
$$

In particular,

$$
\begin{aligned}
& K_{0}^{\prime}(R) \cong Z^{t+1}, \\
& K_{1}^{\prime}(R) \cong Z / Z_{2} \oplus\left(\bigoplus_{j}\left(Z /\left(p_{j}-1\right) Z\right)\right), \\
& K_{2}^{\prime}(R) \cong Z / Z_{2}
\end{aligned}
$$

(see [12, Corollary 10.2 and Corollary 10.8$]$ for the relevant facts on $K_{2}$ ).

Now let $\Lambda$ be a directed set. Let $\left\{S_{\lambda} ; \lambda \in \Lambda\right\}$ be a set of perfect right localizations and let $\left\{\tau_{\lambda}: \lambda \in \Lambda\right\}$ be the associated torsion subcategory of Mod $f$-R. Suppose further that the set $\left\{\tau_{\lambda} ; \lambda \in \Lambda\right\}$ forms a directed family of subcategories with maps $i_{\mu}^{\lambda}: \tau_{\lambda} \rightarrow \tau_{\mu}$ just inclusion functors. Then the $\left\{S_{\lambda} ; \lambda \in \Lambda\right\}$ form a directed family of $R$-modules. It is easy to see that $\tau=\bigcup_{\lambda} \tau_{\lambda}$ is another torsion subcategory of Mod $f-R$ and that the associated localization $S$ of $R$ is isomorphic to $\lim S_{\lambda}$ as a left $R$-module. In particular $S$ is flat as a left $R$-module and is again a perfect right localization of $R$.

THEOREM 2.3. Let $R, S_{\lambda}, S$ and $\tau$ be as described above. Suppose further that each $S_{\lambda}$ is finitely generated as a right $R$-module. Then the long exact $K$-theory localization sequence associated to the localization $S$ of $R$ splits into short exact sequences

$$
0 \rightarrow K_{i}(\tau) \rightarrow K_{i}^{\prime}(R) \rightarrow K_{i}^{\prime}(S) \rightarrow 0
$$

PROOF. We work in the category of $\Lambda$-directed systems of small categories and exact functors. By assumption the set $\left\{\tau_{\lambda} ; \lambda \in \Lambda\right\}$ together with the inclusion functors $i_{\mu}^{\lambda}$ is such a directed system. Similarly the set $\left\{\operatorname{Mod} f-S_{\lambda} ; \lambda \in \Lambda\right\}$ together with the functors $\rho_{\mu}^{\lambda}=-\otimes_{S_{\lambda}} S_{\mu}: \operatorname{Mod} f-S_{\lambda} \rightarrow \operatorname{Mod} f-S_{\mu}$ is a $\Lambda$-directed system. To see this it suffices to observe firstly that $\rho_{\mu}^{\lambda}$ is exact and secondly that $\rho_{\nu}^{\mu} \rho_{\mu}^{\lambda}$ is naturally equivalent to $\rho_{\nu}^{\lambda}$. The first fact follows because for $M \in \operatorname{Mod} f-S_{\lambda}$,

$$
\rho_{\mu}^{\lambda}(M)=M \otimes_{S_{\lambda}} S_{\mu} \cong M \otimes_{R} S_{\lambda} \otimes_{S_{\lambda}} S_{\mu} \cong M \otimes_{R} S_{\mu}
$$


or in other words $\rho_{\mu}$ is naturally equivalent to $-\otimes_{R} S_{\mu}$. The second fact follows similarly. Now since $\tau_{\lambda} \hookrightarrow \operatorname{Mod} f-R \rightarrow \operatorname{Mod} f-S_{\lambda}$ is an exact sequence for each $\lambda$, there is an exact sequence of $\Lambda$-directed systems,

$$
\left\{\tau_{\lambda} ; \lambda \in \Lambda\right\} \hookrightarrow\{\operatorname{Mod} f-R ; \lambda \in \Lambda\} \rightarrow\left\{\operatorname{Mod} f-S_{\lambda} ; \lambda \in \Lambda\right\} .
$$

Taking direct limits then gives a new exact sequence. It is easy to see that $\underline{\lim } \tau_{\lambda}=$ $\tau$, hence since the middle term is again just $\operatorname{Mod} f-R$, we have that $\underset{\lim }{\longrightarrow}\left(\operatorname{Mod} f-S_{\lambda}\right)$ is equivalent to Mod $f-S$. Applying the functor $K_{i}(-)$ to the above exact sequences yields a $\Lambda$-directed system of abelian groups,

$$
0 \rightarrow\left\{K_{i}\left(\tau_{\lambda}\right) ; \lambda \in \Lambda\right\} \rightarrow\left\{K_{i}^{\prime}(R) ; \lambda \in \Lambda\right\} \rightarrow\left\{K_{i}^{\prime}\left(S_{\lambda}\right) ; \lambda \in \Lambda\right\} \rightarrow 0 .
$$

Taking direct limits gies an exact sequence

$$
0 \rightarrow \underline{\lim } K_{i}\left(\tau_{\lambda}\right) \rightarrow \lim _{\longrightarrow} K_{i}^{\prime}(R) \rightarrow \lim _{\longrightarrow} K_{i}^{\prime}\left(S_{\lambda}\right) \rightarrow 0 .
$$

Now using the fact that $\underset{\lim }{\longrightarrow}$ commutes with $K_{i}(-)[13, \S 2]$ we have

$$
\lim _{\longrightarrow} K_{i}\left(\tau_{\lambda}\right) \cong K_{i}\left(\lim _{\lambda} \tau_{\lambda}\right) \cong K_{i}(\tau)
$$

and

$$
\lim _{\longrightarrow} K_{i}\left(S_{\lambda}\right) \cong K_{i}\left(\lim _{\longrightarrow}\left(\operatorname{Mod} f-S_{\lambda}\right)\right) \cong K_{i}(\operatorname{Mod} f-S) .
$$

Hence we have short exact sequences for each $i \geq 0$,

$$
0 \rightarrow K_{i}(\tau) \rightarrow K_{i}^{\prime}(R) \rightarrow K_{i}^{\prime}(S) \rightarrow 0 .
$$

3. Embedding HNP rings in Dedekind domains. In this section we investigate closely the Dedekind prime rings contained between a given HNP ring $R$ and its quotient ring. In particular we show that $R$ is always contained in a Dedekind prime ring which is a directed union of subrings finitely generated as right $R$-modules. We call such a ring a Dedekind closure of $R$. At the same time we prove some results of independent interest. We identify those overrings of $R$ which are Dedekind prime rings and we use this result to say precisely when $R$ is a finite intersection of Dedekind prime rings. Some of these results are hinted at or stated without proof in [17 or 18]. However, to our knowledge, they do not appear anywhere in the literature.

For the readers convenience, we recall some of the well-known properties of HNP rings, as found in $[\mathbf{2}, \mathbf{3}, \mathbf{6}, \mathbf{1 5}]$. If $R$ is an HNP ring, then $R / I$ is artinian for all essential one-sided ideals $I$ of $R$. Hence all finitely generated singular $R$-modules have finite length. Also, it follows that $R$ has right (and left) Krull dimension at most one in the sense of [8]. For any module $A$, we define submodules $\operatorname{soc}^{0}(A) \leq \operatorname{soc}^{1}(A) \leq$ ... by setting $\operatorname{soc}^{0}(A)=0$ and $\operatorname{soc}^{n}(A) / \operatorname{soc}^{n-1}(A)=\operatorname{soc}\left(A / \operatorname{soc}^{n-1}(A)\right)$. In particular, if $A$ is a singular module, then $A=\bigcup_{n=1}^{\infty} \operatorname{soc}^{n}(A)$. For any $R$-module $A$, we use $E_{R}(A)$ to denote the injective hull of $A$, dropping the subscript where no ambiguity can occur.

The right order of an ideal $I$ of $R$ is the ring $O_{r}(I)=\{x \in Q \mid I x \leq I\}$, where $Q$ is the classical quotient ring of $R$. The left order $O_{l}(I)$ is defined similarly. A cycle of idempotent maximal ideals of $R$ is any finite ordered set $\left\{M_{1}, \ldots, M_{n}\right\}$ of distinct idempotent maximal ideals such that $O_{r}\left(M_{1}\right)=O_{l}\left(M_{2}\right), O_{r}\left(M_{2}\right)=$ $O_{l}\left(M_{3}\right), \ldots, O_{r}\left(M_{n}\right)=O_{l}\left(M_{1}\right)$. Stafford and Warfield have recently found an example of an HNP ring containing a cycle of length $p$ for all primes $p[\mathbf{1 8}]$. 
For any set $\Delta$ of simple left $R$-modules, the localization $S$ with respect to $\Delta$ is defined to be

$$
S=\{q \in Q \mid q \in R \text { or the composition factors of } R q+R / R \text { lie in } \Delta\} .
$$

Then $S$ is, in the language of [19], the perfect left localization of $R$ with respect to the torsion theory generated by the members of $\Delta[\mathbf{1 9}$, VII-3, IX and XI]. Such rings were studied by Kuzmanovich in [11] and by Goodearl in [4]. In the language of the latter paper $S$ is the localization of $R$ at the set of maximal left ideals $I$ such that $R / I \notin \Delta$. It is shown in [4] that any ring $T$ contained between $R$ and $Q$ is the localization with respect to the set of simple $R$-modules that occur as subfactors of $T / R$. This then gives a 1-1 order-preserving correspondence between sets of (isomorphism classes of) simple left $R$-modules and subrings of $Q$ containing $R$ [4, Theorem 5]. It is easy to see that if $M$ is an idempotent maximal ideal of $R$, then $O_{r}(M)$ is precisely the localization with respect to the simple left $R$-module annihilated by $M$.

Goodearl's result of course also applies on the right, so rings contained between $R$ and $Q$ are also localizations with respect to a set of simple right $R$-modules (defined analogously). This is the point of view needed to apply Theorems 2.1 and 2.3. However, it is the set of torsion left modules that is most natural to describe, so we will do a certain amount of switching from side to side. The relation between these two sets of simple modules is given in Lemma 4.1 (one is just the dual of the other in the sense described below).

For our purposes it will often be more convenient to work with simple right or left $R$-modules rather than maximal ideals of $R$. There are a number of natural operations on the sets of right and left simple $R$-modules which help clarify the results of [6 and 7] and prove to be useful tools. Firstly there are two operations which take simple right modules to simple left modules and vice versa.

(1) The dual: $V^{\dagger}=\operatorname{Ext}_{R}^{1}(V, R)$. The duality functor $\operatorname{Ext}_{R}^{1}(-, R)$ is in fact defined on the category of all torsion $R$-modules and has many useful properties (see [21] for more details).

(2) The "opposite" (defined only on unfaithful simple modules): we define $V^{\text {op }}$ to be the $R$-module $\operatorname{Hom}_{R / M}(V, R / M)$ if $V$ is an unfaithful module with annihilator $M$. Of course $V^{\text {op }}$ is just the simple right module whose annihilator is the annihilator of the simple left module $V$ and vice versa.

Combining these two operations, yields maps from simple left modules to simple left modules and from simple right modules to simple right modules.

(3) "Upper shriek": $V^{!}=\left(V^{\dagger}\right)^{\text {op }}$ (defined on all simple modules whose dual is unfaithful).

(4) "Lower shriek": $V_{!}=\left(V^{\text {op }}\right)^{\dagger}$ (defined on all unfaithful simple modules).

We can now record some basic facts about these operations.

PROPOSITION 3.1. Let $R$ be an HNP ring and let $V$ be a simple (left or right) $R$-module. Then

(a) $V^{\dagger \dagger} \cong V$.

(b) For $V$ unfaithful, $\left(V^{\mathrm{op}}\right)^{\mathrm{op}} \cong V$.

(c) For $V$ unfaithful, $\left(V_{!}\right)^{!} \cong V$.

(d) For $V$ such that $V^{\dagger}$ is unfaithful, $\left(V^{!}\right)_{!} \cong V$. 
PROOF. Part (a) is a well-known property of the duality functor proved in [21]. The other parts are then clear.

We now wish to reinterpret some of the main results of [6] into our new terminology.

PROPOSITION 3.2. Let $R$ be an HNP ring and let $V$ be an unfaithful simple left $R$-module with annihilator $M$.

(1) If $M$ is invertible, then $V_{!} \cong V$.

(2) If $M$ is idempotent and $U$ is another unfaithful simple left $R$-module with annihilator $N$, then $U \cong V_{!}$if and only if $N$ is idempotent and $O_{l}(M)=O_{r}(N)$.

(3) If $U$ is a simple left $R$-module, then $\operatorname{Ext}_{R}^{1}(U, V) \neq 0$ if and only if $U \cong V_{!}$.

Proof. Let $Q$ be the classical quotient ring of $R$ and let $M^{*}=\{x \in Q \mid x M \in$ $R\}$. Then it is clear from the definition that $V_{!}$is a simple submodule of the left $R$-module $M^{*} / R$. If $M$ is invertible $M M^{*}=R$, so $V_{!} \cong V$. Part (3) then follows in this case from [6, Proposition 1]. On the other hand, if $M$ is not invertible, then $M^{*}=O_{l}(M)$ and the rest of the proposition follows from [6, Theorems 7 and 8].

We define a cycle of simple left $R$-modules to be a set $\left\{U_{1}, \ldots, U_{n}\right\}$ of simple left $R$-modules such that $U_{i} \cong\left(U_{i-1}\right)$ ! for all $i=2, \ldots, n$ and $U_{1} \cong\left(U_{n}\right)$ !. Then by part (2) of the above proposition, the annihilators of the $U_{i}$ form a cycle of idempotent maximal ideals.

Since it will be fundamental to what follows we now state one of the main theorems of $[6]$.

THEOREM 3.3. Let $R$ be an HNP ring and let $U$ be an unfaithful simple left $R$-module. Set $E_{i}=\operatorname{soc}^{i}\left(E_{R}(U)\right) / \operatorname{soc}^{i-1}\left(E_{R}(U)\right)$. Then one of the following situations occurs.

(1) $E_{i} \cong U$ for all $i$. (In this case $\operatorname{ann}_{R} U$ is an invertible ideal.)

(2) There exists a cycle $\left\{U=U_{1}, U_{2}, \ldots, U_{n}\right\}$ of unfaithful simple left $R$-modules such that $E_{i} \cong U_{k}$ whenever $k \in\{1, \ldots, n\}$ and $i \equiv k(\bmod n)$. (In this case the ideals $\operatorname{ann}_{R} U_{i}$ form a cycle of idempotent maximal ideals).

(3) There exists an $n$ such that $E_{i}$ is an unfaithful simple left $R$-module for $i=1, \ldots, n$ and $E_{n+1}$ is a faithful simple left $R$-module. Furthermore, $E_{i+1} \cong\left(E_{i}\right)$ ! for all $i=1, \ldots, n$. (In this case $\operatorname{ann}_{R} U$ is an idempotent maximal ideal not contained in any cycle.)

We define a simple left $R$-module to be of type 1, 2 or 3 according to which of the above situations occur. Notice that the idempotent maximal ideals are the annihilators of the type 2 and 3 simple left $R$-modules. Hence $R$ is a Dedekind prime ring if and only if all simple $R$-modules are of type 1 .

There is a natural directed graph $\Gamma$ associated to the set $\Phi$ of unfaithful simple left $R$-modules. The vertices of the graph $\Gamma$ are the isomorphism classes of unfaithful simple left $R$-modules and the vertex $\underline{V}$ is linked by an arrow to $\underline{U}$ if and only if $U=V_{!}$. This graph is of course equivalent to the link graph of prime ideals described in [10]. Its connected components are either cycles or strings (if we consider a vertex linked to itself as a trivial cycle and a vertex with no edges coming out of it as a trivial string). Notice that all the strings are finite by [6, Corollary 21].

We define an equivalence relation on unfaithful simple left $R$-modules via $U \sim V$ iff $\underline{U}$ and $\underline{V}$ lie in the same connected component of $\Gamma$. For $\Delta$ a subset of $\Phi$, we 
define

$$
\bar{\Delta}=\{U \in \Phi \mid U \sim V \text { for some } V \in \Delta\} .
$$

We will be interested in the following types of subset of $\Phi$. A full, deleted subset of $\Phi$ is a subset $\Delta$ of type 2 and 3 modules such that $\bar{\Delta} \backslash \Delta$ consists of precisely one element of each cycle in $\bar{\Delta}$. A complete deleted subset of $\Phi$ is a full deleted subset $\Delta$ such that $\bar{\Delta}$ contains all the type 2 or 3 modules.

We now proceed to construct the "Dedekind closure" of an arbitrary HNP ring as described in the introduction. There are of course two different approaches to showing that a ring is a Dedekind prime ring. One is to show that it has no idempotent maximal ideals and the other is to show it only has type 1 unfaithful simple modules. Here we take the module theoretic approach for two reasons: firstly because it fits in better with the techniques of the rest of the paper but mainly because simple modules are slightly easier to keep track of under this general localization than are maximal ideals.

We first state explicitly some results from [19] that will be used frequently in the following.

LEMMA 3.4. Let $R$ be a ring and let $S$ be a perfect left localization of $R$. Let $M$ be an $S$-torsion-free left $R$-module. Then as $R$-modules, $E_{R}(M) \cong E_{R}\left(S \otimes_{R} M\right) \cong$ $E_{S}\left(S \otimes_{R} M\right)$. Furthermore, as an $R$-module, $S \otimes_{R} M$ is isomorphic to the largest submodule $N$ of $E_{R}(M)$ such that $N / M$ is $S$-torsion.

ProOF. [19, IX.2.3].

LEMMA 3.5. Let $R$ be an HNP ring, let $\Delta$ be a set of simple left $R$-modules and let $S$ be the localization of $R$ with respect to $\Delta$. Let $U$ be a simple left $R$-module such that $U \notin \Delta$.

(1) If $U$ is a type $1 R$-module, then $S \otimes_{R} U \cong U$ as $R$-modules and $S \otimes_{R} U$ is a type 1 simple $S$-module.

(2) If $\left\{U=U_{1}, U_{2}, \ldots, U_{n}\right\}$ is a cycle of simple left $R$-modules and $U_{i} \in \Delta$ for $i=2, \ldots, n$, then $S \otimes_{R} U \cong \operatorname{soc}^{n}\left(E_{R}(U)\right)$ and $S \otimes_{R} U$ is a simple left $S$-module of type 1.

(3) If $U$ is a type $3 R$-module, then $S \otimes_{R} U$ is either a faithful or a type 3 unfaithful simple $S$-module.

ProOF. These follow from Lemma 3.4 and [6, Theorem 22].

We shall use the following result later in $\S 5$.

Proposition 3.6. Let $R$ be an HNP ring. Let $\Delta$ be a full deleted set of unfaithful simple left $R$-modules and let $S$ be the localization of $R$ with respect to $\Delta$. Let $P^{\prime}$ be a nonzero idempotent maximal ideal of $S$ and let $P=P^{\prime} \cap R$. Then $P$ is an idempotent maximal ideal of $R$ such that $P V \neq 0$ for all $V \in \bar{\Delta}$. Furthermore $P^{\prime}=S P=P S=S P S$.

PROOF. Let $V^{\prime}$ be an unfaithful simple left $S$-module annihilated by the nonzero idempotent maximal ideal $P^{\prime}$. Then $V=\operatorname{soc}_{R}\left(V^{\prime}\right)$ is an unfaithful simple $R$-module and $S \otimes_{R} V \cong V^{\prime}$. Now since $V^{\prime}$ is a type 2 or 3 simple $S$-module, it follows from Lemma 3.5 that $V$ is a type 2 or 3 simple $R$-module such that $V \notin \bar{\Delta}$. Thus since $\Delta$ is a full deleted set, $V^{!} \cong \operatorname{soc}^{2}\left(E_{R}(V)\right) / V \notin \Delta$. Hence by Lemma 3.4 , $V^{\prime}=V$. Thus if $P=\operatorname{ann}_{R} V$, it is clear that $P=P^{\prime} \cap R$. Further $P$ is idempotent 
because $V$ is type 2 or 3 . But now since $S$ is both a right and left localization of $R$ by [4], we always have, for any right (resp. left) ideal of $R$, that $I=(I \cap R) S$ (resp. $I=S(I \cap R)$ ), whence it follows that $P^{\prime}=S P=P S=S P S$.

THEOREM 3.7. Let $R$ be an HNP ring, let $\Delta$ be a complete deleted set of unfaithful simple left $R$-modules and let $S$ be the localization of $R$ with respect to $\Delta$. Then $S$ is a Dedekind prime ring.

PROOF. This result is immediate from the proposition and the definition of a complete deleted set.

DEFINITION. A localization $S$ of an HNP ring $R$ with respect to a complete deleted set of unfaithful simple modules will be called a Dedekind closure of $R$.

These Dedekind closures $S$ have two other interesting properties: firstly, no ring strictly contained between $R$ and $S$ is also a Dedekind prime ring and secondly, $S$ is the directed union of subrings right equivalent to $R$.

In order to prove the first statement, we prove a slightly more general result which identifies exactly which rings containing $R$ are Dedekind prime rings. In particular this enables us to state precisely which HNP are finite intersections of Dedekind prime rings.

THEOREM 3.8. Let $R$ be an HNP ring, let $\Delta$ be a set of simple left $R$-modules and let $S$ be the localization of $R$ with respect to $\Delta$. Then $S$ is a Dedekind prime ring if and only if the following two conditions hold.

(1) Each cycle of unfaithful simple left $R$-modules contains at most one member not in $\Delta$.

(2) For any complete string $\Sigma=\left\{U_{1}, \ldots, U_{n}\right\}$ of type 3 unfaithful simple left $R$ modules, either $\Sigma \leq \Delta$ or there exists an $i$ between 1 and $n$ such that $\left\{U_{1}, \ldots, U_{i-1}\right.$, $\left.U_{i+1}, \ldots, U_{n}, U_{n}^{!}\right\} \leq \Delta$.

PROOF. First suppose that the two conditions are satisfied. Let $V^{\prime}$ be an unfaithful simple left $S$-module and let $V=\operatorname{soc}_{R}\left(V^{\prime}\right)$. Suppose that $V^{\prime}$ is a type 2 or $3 S$-module. Then $V$ must be an unfaithful type 2 or $3 R$-module by Lemma 3.5. Because of condition (1) and Lemma 3.5 part (2), $V$ cannot be type 2 either. Hence $V$ is type 3 and $V$ belongs to a string $\left\{V=V_{1}, \ldots, V_{t}\right\}$ where $V_{i} \cong V_{i-1}^{!}$and $V_{t}^{!}$ is faithful. But by condition (2) and Lemma $3.4, V^{\prime} \cong S \otimes_{R} V$ contains $V_{t}^{!}$as an $R$-subfactor. Thus $V^{\prime}$ is a faithful $R$-module and hence is a faithful $S$-module also, contradicting our assumption.

On the other hand, assume that $S$ is a Dedekind prime ring. Suppose we have unfaithful simple left $R$-modules $U=U_{1}, \ldots, U_{t}$ such that $U_{i} \cong U_{i-1}^{!}$for $i=$ $2, \ldots, n$, and neither $U$ nor $U_{t}^{!}$lie in $\Delta$. Then by 3.2, 3.3 and 3.4,S $S \otimes_{R} U$ is an unfaithful simple $S$-module. But $U_{t}^{!}$is a subfactor of $E_{R}(U)$ so by $3.4, S \otimes_{R} U_{t}^{!}$is a subfactor of $E_{S}\left(S \otimes_{R} U\right)$. Since $S$ is Dedekind, we must have $S \otimes_{R} U \cong S \otimes_{R} U_{t}^{!}$. But this implies that $U \cong U_{t}^{!}$, whence both conditions (1) and (2) must hold.

COROLLARY 3.9. Let $R$ be an HNP ring. Let $\Delta$ be a complete deleted set of unfaithful simple left $R$-modules and let $S$ be the localization of $R$ with respect to $\Delta$. If $T$ is a Dedekind prime ring contained between $R$ and $S$ then $T=S$.

PROOF. If $T$ were strictly contained in $S$, then it would be localization with respect to some proper subset of $\Delta$. Hence by Theorem 3.8 it could not be a Dedekind prime ring. 
If $R$ is an HNP ring with quotient ring $Q$ then it is easy to see that $R$ is the intersection of all the Dedekind prime rings between $R$ and $Q$. For the localization of $R$ with respect to all but one simple $R$-module is always a Dedekind prime ring and $R$ is easily seen to be the intersection of these localizations. If $R$ has finitely many idempotent maximal ideals, then it is well known that $R$ is a finite intersection of Dedekind prime rings. On the other hand in [18] two examples of HNP rings with infinitely many idempotent ideals are given, one of which is, and one of which is not an intersection of finitely many Dedekind prime rings. The exact conditions for this to occur are the following.

THEOREM 3.10. Let $R$ be an HNP ring with classical ring of quotients $Q$. Then $R$ is the intersection of finitely many Dedekind domains contained between $R$ and $Q$ if and only if there is a bound on the lengths of the connected components of the link graph $\Gamma$.

PROOF. For any complete string $\left\{U_{1}, \ldots, U_{n}\right\}$ of type 3 unfaithful simple left $R$-modules, call $\left\{U_{1}, \ldots, U_{n}, U_{n}^{!}\right\}$the corresponding extended string. Let $T$ be the localization of $R$ with respect to a set of simple left $R$-modules $\Delta$. If $T$ is a Dedekind prime ring, then there is at most one member from each cycle or extended string not contained in $\Delta$. Hence if there exists a cycle or extended string of cardinality $n$, then any intersection of less than $n$ Dedekind pime rings containing $R$ must strictly contain $R$.

On the other hand, if all cycles and extended strings have cardinality less than some fixed integer $t$, then it is clear from Theorem 3.8 that we can find $t$ sets of simple left $R$-modules whose intersection is empty and such that the corresponding localization is Dedekind.

4. $K_{i}(R)$ for HNP rings. In this section we apply the results of $\S 1$ to the case where $R$ is an HNP ring and $S$ is a ring contained between $R$ and its quotient ring. We identify $K_{i}()$ as the direct sum of the $K_{i}\left(\operatorname{End}_{R} V\right)$ where $V$ is an $S$-torsion simple module. In particular we look at the situation where $S$ is a Dedekind closure of $R$ (as defined after Theorem 3.7). The only problem in this situation is that the left torsion theory associated to $S$ is easier and more natural to identify than the right torsion theory. We show that, since one torsion theory is the dual of the other, their $K_{i}$-groups are in fact the same.

LEMMA 4.1. Let $R$ be an HNP ring and let $S$ be a ring contained between $R$ and its quotient ring $Q$. Let $\Delta_{l}\left(\right.$ resp. $\left.\Delta_{r}\right)$ be the set of $S$-torsion simple left (resp. right) $R$-modules. Then

$$
\Delta_{r}=\Delta_{l}^{\dagger}=\left\{V^{\dagger} \mid V \in \Delta_{l}\right\}
$$

PROOF. It is shown in [4], that $S$ is always the localization with respect to the $S$-torsion simple modules. Furthermore it is shown that a simple $R$-module $V$ is $S$-torsion if and only if it occurs as a subquotient of $S / R[\mathbf{4}$, Theorem 5]. Let $V \in \Delta_{l}$, and suppose $V \cong R / I$ for some left ideal $I$. Then by the definition of localization with respect to a set of simple modules,

$$
I^{*}=\{q \in Q \mid I q \leq R\} \leq S .
$$


So $V^{\dagger} \cong I^{*} / R$ is a right $R$-submodule of $S / R$. Hence $V^{\dagger} \in \Delta_{r}$ and $\Delta_{l}^{\dagger} \leq \Delta_{r}$. The analogous result on the other side gives that $\Delta_{r}^{\dagger} \leq \Delta_{l}$ and taking duals gives $\Delta_{r} \leq \Delta_{l}^{\dagger}$, as required.

LEMMA 4.2. Let $R$ be an HNP ring and let $V$ be a simple $R$-module. Then there is a natural isomorphism, $\operatorname{End}_{R}(V) \cong \operatorname{End}_{R}\left(V^{\dagger}\right)$.

Proof. Suppose $V$ is a simple left module and $I$ is a left ideal such that $R / I \cong$ $V$. Then $V^{\dagger} \cong I^{*} / R$ and it is easy to check that $\operatorname{End}_{R}\left(V^{\dagger}\right) \cong \mathbb{Q}(I) / I \cong \operatorname{End}_{R}(V)$.

THEOREM 4.3. Let $R$ be an HNP ring and let $M_{1}, \ldots, M_{t}$ be idempotent maximal ideals of $R$ such that $M=\left(M_{1} \cap \cdots \cap M_{t}\right)^{t}$ is idempotent. Then for all $i \geq 0$,

$$
K_{i}(R) \cong K_{i}\left(O_{r}(M)\right) \oplus K_{i}\left(R /\left(M_{1} \cap \cdots \cap M_{t}\right)\right) .
$$

ProOF. Let $V_{1}, \ldots, V_{t}$ be simple left $R$-modules with $M_{j} V_{j}=0$ for $j=1, \ldots, t$. Then $O_{r}(M)$ is the left localization of $R$ with respect to $\left\{V_{1}, \ldots, V_{t}\right\}$. Hence the modules of the class $\tau$ of finitely generated right $O_{r}(M)$-torsion modules have composition series with factors in $\left\{V_{1}^{\dagger}, \ldots, V_{t}^{\dagger}\right\}$. Let $D_{j}=\operatorname{End}_{R}\left(V_{j}\right)=$ $\operatorname{End}_{R}\left(V_{j}^{\dagger}\right)$. Then by [13, Corollary 5.2 and p. 104], $K_{i}(\tau) \cong \bigoplus_{1 \leq j \leq t} K_{i}\left(D_{j}^{\text {op }}\right) \cong$ $\bigoplus_{1 \leq j \leq t} K_{i}\left(D_{j}\right)$. Now $O_{r}(M)$ is finitely generated as a right $R$-module by [15, Theorem 4.4], so by Corollary 2.2,

$$
K_{i}(R) \cong K_{i}\left(O_{r}(M)\right) \oplus\left(\bigoplus_{1 \leq j \leq t} K_{i}\left(D_{j}\right)\right)
$$

But of course $R / M_{j}$ is a matrix ring over $D_{j}$, so

$$
\left.\bigoplus_{1 \leq j \leq t} K_{i}\left(D_{j}\right) \cong K_{i}\left(R / M_{1} \cap \cdots \cap M_{t}\right)\right) .
$$

In particular this occurs when $M$ is a minimal idempotent ideal and $O_{r}(M)$ is a Dedekind prime ring right equivalent to $R$. We state this case in a slightly different form.

THEOREM 4.4. Let $R$ be an HNP ring, let $S$ be a Dedekind prime ring right equivalent to $R$ and let $\tau$ be the category of finitely generated $S$-torsion $R$-modules. Then the long exact localization sequence splits into short exact sequences,

$$
0 \rightarrow K_{i}^{\prime}(\tau) \rightarrow K_{i}(R) \rightarrow K_{i}(S) \rightarrow 0 .
$$

Thus,

$$
K_{i}(R) \cong K_{i}(S) \oplus\left(\bigoplus K_{i}(\operatorname{End}(V))\right)
$$

where the direct sum is over isomorphism classes of simple modules of $\tau$. In particular,

$$
K_{0}(R) \cong K_{0}(S) \oplus Z^{t}
$$

where $t$ equals the number of idempotent maximal ideals of $R$ minus the number of cycles.

PROOF. Since $S$ is right equivalent to $R$, it is certainly finitely generated as a right $R$-module. Since $S$ is also flat as a left $R$-module the first statement follows 
from Theorem 2.1. The second statement follows from the same identifications used in the proof of Theorem 4.3. The final statement follows from the fact that $t$ is precisely the number of isomorphism classes of simple modules in any complete deleted set of unfaithful simple left $R$-modules and $S$ must be a localization with respect to such a set.

We denote by $\mathbb{\Omega}_{R}(I)$ the idealizer in $R$ of the right ideal $I$ as defined in [15].

THEOREM 4.5. Let $S$ be an HNP ring and let $I$ be a semimaximal right ideal of $S$ with $S I=I$. Then

$$
K_{i}\left(\mathbb{\vartheta}_{S}(I)\right) \cong K_{i}(S) \oplus K_{i}\left(\mathbb{\Downarrow}_{S}(I) / I\right) .
$$

We now give a more general version of the previous theorem for localizations of $R$ which are direct limits of rings right equivalent to $R$. For this we use Theorem 2.3 so of course we lose the splitting of the exact sequence. It will however be shown in $\S 5$ that for $i=0$, this sequence does in fact split. We do not know whether this is true for $i \geq 1$.

THEOREM 4.6. Let $R$ be an HNP ring and let $\Delta$ be a set of unfaithful simple left $R$-modules such that (1) $\operatorname{ann}_{R} V$ is idempotent for all $V \in \Delta$ and (2) $\Delta$ contains no cycles. Let $S$ be the localization of $R$ with respect to $\Delta$. Then for all $i \geq 0$, there are exact sequences,

$$
0 \rightarrow \bigoplus_{V \in \Delta} K_{i}\left(\operatorname{End}_{R} V\right) \rightarrow K_{i}(R) \rightarrow K_{i}(S) \rightarrow 0 .
$$

ProOF. Let $\Lambda$ be the set of finite subsets of $\Delta$. For each $\lambda \in \Lambda$, define $S_{\lambda}$ to be the localization of $R$ with respect to the simple modules in $\lambda$. The assumption on $\Delta$ implies that the intersection $I$ of the annihilators of the elements of $\lambda$ is not contained in any invertible ideal [3, Proposition 4.3]. Hence for some $t, I^{t}$ is idempotent by [3, Proposition 4.5] and hence $S=O_{r}\left(I^{t}\right)$. Thus each $S_{\lambda}$ is finitely generated as a right $R$-module. It is clear that $\Lambda$ is a partially ordered, directed set under inclusion and that $S \cong \underset{\lim }{\longrightarrow} S_{\lambda}$. Hence we may apply Theorem 2.3, yielding an exact sequence

$$
0 \rightarrow K_{i}(\tau) \rightarrow K_{i}(R) \rightarrow K_{i}(S) \rightarrow 0
$$

where $\tau$ is the torsion class of finitely generated $S$-torsion right $R$-modules. Since all objects of $\tau$ have composition series with factors in $\Delta^{\dagger}$, we have by $[\mathbf{1 3}$, Corollary 5.2 and p. 104] and Lemmas 4.1 and 4.2 , that $K_{i}(\tau) \cong \bigoplus_{V \in \Delta^{\dagger}} K_{i}\left(\operatorname{End}_{R} V\right) \cong$ $\bigoplus_{V \in \Delta} K_{i}\left(\operatorname{End}_{R} V\right)$.

Again the above theorem applies particularly to the case when $\Delta$ is a complete deleted set and $S$ is a Dedekind closure of $R$. In particular we will be interested in the next section with the case $i=0$, so we state explicitly the result in this case.

COROLLARY 4.7. Let $R$ be an HNP ring with infinitely many idempotent maximal ideals and let $S$ be a Dedekind closure of $R$. Then there is a natural exact sequence

$$
0 \rightarrow Z^{(\alpha)} \rightarrow K_{0}(R) \rightarrow K_{0}(S) \rightarrow 0
$$

where $\alpha$ is the cardinality of the set of idempotent maximal ideals.

EXAMPLE. We now consider $K_{0}(R)$ for the examples of HNP rings with infinitely many idempotent maximal ideals given in [18]. In each case it can fairly 
easily be seen that $R$ has a natural Dedekind closure $S$ which is a localization of the polynomial ring $D[X]$ over some division ring $D$. In particular $K_{0}(S) \cong Z$. Thus by Corollary $4.7, K_{0}(R)$ is a free abelian group whose rank is the cardinality of the set of idempotent maximal ideals. For the rings $H$ defined in $\S \S 2$ and 3 of [18] this cardinality is $\aleph_{0}$ while for the ring $R_{1}$ described in $\S 4$, the cardinality is just the cardinality of the given index set $\Lambda$. Similar calculations were done by Stafford and Warfield in their paper [18, Corollary 4.10]. However their technique required the special fact that these rings are direct limits of HNP subrings with finitely many idempotent ideals.

5. Class groups and $H_{0}(R)$ for HNP rings. A classical result in the theory of Dedekind domains states that if $R$ is a Dedekind domain, then $K_{0}(R) \cong Z \oplus \mathrm{Cl}(R)$ where the class group $\mathrm{Cl}(R)$ is the group of isomorphism classes of ideals of $R$ under multiplication. For Dedekind prime rings there is a natural analogue of this isomorphism, though the definition of class group is much less natural. For a Dedekind prime ring $R$ with quotient ring $Q$ we define $\mathrm{Cl}(R)=\left\{[I] \in K_{0}(R) \mid I\right.$ is a finitely generated projective $R$-module such that length $\left.\left(I \otimes_{R} Q\right)=\operatorname{length}(Q)\right\}$ where the operation is now $[I] \cdot[J]=[I]+[J]-[R]$. Then it is easy to show using the results of [2] that this operation makes $\mathrm{Cl}(R)$ into a group and that $K_{0}(R) \cong Z \oplus \mathrm{Cl}(R)$ (this result appears in [14] for maximal orders over Dedekind domains but does not seem to be explicitly stated in the literature for general Dedekind prime rings).

For a nonmaximal hereditary order $R$ over a Dedekind domain, the class group is defined in [14] to be the group of stable isomorphism classes of "locally free" right ideals of $R$. This definition is generalized below to the case where $R$ is an HNP ring and it is shown that $K_{0}(R) \cong F \oplus \mathrm{Cl}(R)$ where $F$ is a free abelian group whose rank depends on the number of idempotent maximal ideals of $R$.

This decomposition is closely related to the localization exact sequence associated to a Dedekind closure $S$ of $R$. It is shown that there is a natural isomorphism $\mathrm{Cl}(R) \cong \mathrm{Cl}(S)$ (generalizing a result of Jacobinski's for hereditary orders over Dedekind domains [14, Theorem 40.16]). Hence for a suitable decomposition $F \cong$ $F_{0} \oplus R$, the map $K_{0}(R)$ to $K_{0}(S)$ described in Corollary 4.7 is just the projection of $F_{0} \oplus R \oplus \mathrm{Cl}(R)$ onto $R \oplus \mathrm{Cl}(R)$. In particular this shows that this exact sequence always splits.

The crucial concept needed for this analysis is that of a generalized rank function or state. A state is a group homomorphism $s: K_{0}(R) \rightarrow \mathbf{R}$ ( $\mathbf{R}$ denoting the real numbers) such that $s([R])=1$ and for every finitely generated projective $R$-module $A, s([A]) \geq 0$. The reader is referred to [5 and $\mathbf{7}]$ for more details. We shall mostly be concerned with the states that occur in the following fashion. If $R$ is an HNP ring and $P$ is a nonzero prime ideal, then $R / P$ is a simple artinian ring and we may define, for any finitely generated projective (right) $R$-module $A$,

$$
r_{P}(A)=\operatorname{length}\left(A \otimes_{R} R / P\right) / \operatorname{length}(R / P) .
$$

Since $r_{P}$ is additive on direct sums and $r_{P}([R])=1$, we see that $r_{P}$ induces a state $s_{P} \in \operatorname{St}(R)$ such that $s_{P}([A]-[B])=r_{P}(A)-r_{P}(B)$ for any finitely generated projective $R$-modules $A$ and $B$. Similarly the usual normalized rank function given by $r_{0}(A)=\operatorname{length}\left(A \otimes_{R} Q\right) /$ length $(Q)$ (where $Q$ is the quotient ring of $R$ ) induces a state which we will denote by $s_{0}$. 
Each $x \in K_{0}(R)$ now defines a map from $\operatorname{Spec} R$ to $Q$ given by $\rho_{x}(P)=s_{P}(x)$ for all $P \in \operatorname{Spec} R$. This map tuns out to be locally constant when $\operatorname{Spec} R$ is equipped with the patch topology [7]. Hence we obtain a homomorphism $\rho$ from $K_{0}(R)$ to the group of locally constant maps from $\operatorname{Spec} R$ to $Q$ given by $\rho(x)=\rho_{x}$. Following the roughly analogous situation in $[1$, p. 458$]$ for commutative rings, we denote the image of this map by $H_{0}(R)$. We then have a short exact sequence

$$
0 \rightarrow \operatorname{Ker} \rho \rightarrow K_{0}(R) \rightarrow H_{0}(R) \rightarrow 0 .
$$

We now proceed to identify Ker $\rho$ with a "constant rank right ideal class group."

DEFINITION 5.1. For $R$ an HNP ring, define

$$
\mathrm{Cl}(R)=\left\{[R]-[I] \mid I \text { is a right ideal of } R \text { and } r_{P}(I)=1 \text { for all } P \in \operatorname{Spec} R\right\} \text {. }
$$

The following result has also been observed independently by R. B. Warfield. In fact the result is true for any prime Noetherian ring of Krull dimension one and exactly the same proof goes through.

LEMMA 5.2. Let $R$ by an $H N P$ ring and let $\rho: K_{0}(R) \rightarrow H_{0}(R)$ be the map described above. Then $\operatorname{Ker} \rho=\mathrm{Cl}(R)$. In particular $\mathrm{Cl}(R)$ is a subgroup of $K_{0}(R)$.

ProOF. It is immediate from the definition of $\rho$ that if $x \in K_{0}(R)$, then $x \in \operatorname{Ker} \rho$ if and only if $s_{P}(x)=0$ for all $P \in \operatorname{Spec} R$. Thus it is clear that $\mathrm{Cl}(R) \leq \operatorname{Ker} \rho$. On the other hand, let $x$ be an arbitrary element of Ker $\rho$. Then $x=n[R]-[A]$ where $n$ is a positive integer and $A$ is a finitely generated projective $R$-module with $r_{P}(A)=n$ for all $P \in \operatorname{Spec} R$. By [16, Lemma 5.2 and Corollary 5.8] $A \cong R^{(n-1)} \oplus A_{0}$ and hence $x=[R]-\left[A_{0}\right]$ where $r_{P}\left(A_{0}\right)=1$ for all $P \in \operatorname{Spec} R$. But $A_{0}$ is then isomorphic to a right ideal of $R$, so $x \in \mathrm{Cl}(R)$ as required.

We now want to compare the exact sequence $0 \rightarrow \mathrm{Cl}(R) \rightarrow K_{0}(R) \rightarrow H_{0}(R) \rightarrow 0$ with the exact sequences for $K_{0}$ derived in the previous section. We first need to see how $\mathrm{Cl}(-)$ behaves with respect to Dedekind closure. Again we have finite and infinite versions.

Define $K_{0}(R)^{+}=\left\{[A] \in K_{0}(R) \mid A\right.$ is a finitely generated projective $R$-module $\}$.

LEMMA 5.3. Let $R$ be a ring and let $G$ be a subgroup of $K_{0}(R)^{+}$such that for some $x \in K_{0}(R), x-g \in K_{0}(R)^{+}$for all $g \in G$. Then $s(g)=0$ for all $s \in \operatorname{St}(R)$ and all $g \in G$.

ProOF. Since $s(x-g) \geq 0$ and $s(x+g) \geq 0$, it follows that $|s(g)| \leq|s(x)|$ for all $g \in G$. Hence since it is a bounded additive subgroup of $R, s(G)$ must be zero.

LEMMA 5.4. Let $R$ be an HNP ring. Then $\mathrm{Cl}(R)$ is the unique maximal subgroup of $K_{0}(R)$ consisting entirely of elements of the form $[V]$ where $V$ is a cyclic torsion $R$-module.

Proof. Let $x=[R]-[I] \in \mathrm{Cl}(R)$. Since $r_{0}(I)=1, I$ must be an essential right ideal of $R$. Thus $R / I$ is torsion and $x=[R / I]$ is of the required form.

Now let $G$ be a subgroup of $K_{0}(R)$ consisting of elements of the form [ $\left.V\right]$ where $V$ is a cyclic torsion $R$-module. Any element $y$ of $G$ may be expressed in the form $y=[R / J]=[R]-[J]$ where $J$ is a right ideal of $R$. But this implies that $[R]-y \in K_{0}(R)^{+}$for all $y \in G$. Hence by Lemmas 5.2 and $5.3, G$ is contained in $\mathrm{Cl}(R)$. 
LEMMA 5.5. Let $R$ by an HNP ring and let $S$ be a ring contained between $R$ and its quotient ring. Then the functor $-\otimes_{R} S$ induces a map from $\mathrm{Cl}(R)$ to $\mathrm{Cl}(S)$.

Proof. The functor $-\otimes_{R}(S)$ induces a map $\phi: K_{0}(R) \rightarrow K_{0}(S)$. Since $S$ is a flat $R$-module the image of $\mathrm{Cl}(R)$ under $\phi$ again consists of cyclic torsion $S$-modules. So $\phi(\mathrm{Cl}(R)) \leq \mathrm{Cl}(S)$ by Lemma 5.4 .

PROPOSITION 5.6. Let $R$ by an HNP ring and let $S$ be a ring contained between $R$ and its quotient ring such that $S$ is a finitely generated $R$-module. Then the induced map from $\mathrm{Cl}(R)$ to $\mathrm{Cl}(S)$ is an epimorphism.

PROOF. We know from Theorem 2.1 that the induced map $\phi: K_{0}(R) \rightarrow K_{0}(S)$ is split by the map $\theta: K_{0}(S) \rightarrow K_{0}(R)$ induced from the restriction functor. An arbitrary element of $\theta(\mathrm{Cl}(S))$ is of the form $[S]-[J]$ for some right ideal $J$ of $S$. Since $J$ is a finitely generated projective $R$-module, this implies that $[S]-y \in$ $K_{0}(R)^{+}$for all $y \in \theta(\mathrm{Cl}(S))$. So $\theta(\mathrm{Cl}(S)) \leq \mathrm{Cl}(R)$ by Lemma 5.3. Since $\phi \theta$ is the identity, it is clear that $\phi(\mathrm{Cl}(R))=\mathrm{Cl}(S)$.

LEMMA 5.7. Let $R$ be an HNP ring and let $S$ be a ring contained between $R$ and its quotient ring such that $S$ is a finitely generated right $R$-module. Suppose that $M^{\prime}$ is a maximal ideal of $S$ such that $M^{\prime}=M S=S M$ for some maximal ideal $M$ of $R$. Then for any finitely generated projective right $R$-module $A, r_{M}(A)=r_{M^{\prime}}\left(A \otimes_{R} S\right)$.

Proof. The condition that $M^{\prime}=S M=M S$ together with the fact that $S$ is a localization of $R$, insures that $R / M \cong S / M^{\prime}$. Since these rings are their own classical quotient rings,

$$
r_{m}(A)=\frac{\operatorname{length}\left(A \otimes_{R} R / M\right)}{\text { length }(R / M)}=\frac{\operatorname{length}\left(A \otimes_{R} S \otimes_{S} S / M^{\prime}\right)}{\text { length }\left(S / M^{\prime}\right)}=r_{M^{\prime}}\left(A \otimes_{R} S\right) .
$$

THEOREM 5.8. Let $R$ be an HNP ring, let $\Phi$ be a complete deleted set of simple left $R$-modules and let $S$ be the localization of $R$ with respect to $\Phi$. Then the function $-\otimes_{R} S$ induces an epimorphism from $\mathrm{Cl}(R)$ to $\mathrm{Cl}(S)$.

PROOF. It is clear from the definition of $\mathrm{Cl}(S)$ that it is enough to show that for all finitely generated projective right $S$-modules $I^{\prime}$ such that $[S]-\left[I^{\prime}\right] \in \mathrm{Cl}(S)$, there exists a finitely generated projective right $R$-module $I$ with $[R]-[I] \in \mathrm{Cl}(S)$ and $\left[I \otimes_{R} S\right]=\left[I^{\prime}\right]$. So let $I^{\prime}$ be a finitely generated projective right $S$-module with $[S]-\left[I^{\prime}\right] \in \mathrm{Cl}(S)$. Pick a finitely generated $R$-submodule $I$ of $I^{\prime}$ such that $I^{\prime}=I S \cong I \otimes_{R} S$. Then $I$ is projective and $r_{0}(I)=1$ but $[R]-[I]$ may not lie in $\mathrm{Cl}(R)$. Now by $[\mathbf{7}, 4.10]$ the map $\rho_{[I]}: \operatorname{Spec} R \rightarrow Q$ is locally constant when $\operatorname{Spec} R$ is given the patch topology, so in particular there is an open set $U$ containing (0) such that $r_{P}(I)=1$ for all $P \in U$. However the open sets of $\operatorname{Spec} R$ containing (0) are just complements of finite sets. Hence there are only finitely many prime ideals $P$ of $R$ such that $r_{P}(I) \neq 1$ (and these must be idempotent by $[7,5.1]$ ). Let $\Delta$ be a minimal full deleted subset of $\Phi$ such that $\bar{\Delta}$ contains all the simple modules annihilated by these primes $P$. By the comments following Theorem $3.3, \Delta$ must be finite and hence, since $\Delta$ contains no cycles, the localization $T$ with respect to $\Delta$ is finitely generated as a right $R$-module. By Proposition 3.6, if $M$ is an idempotent ideal of $T$, then $M=T(M \cap R)=(M \cap R) T$ and $M \cap R$ is a maximal ideal of $R$ such that $(M \cap R) V \neq 0$ for all $V \in \bar{\Delta}$. Hence $r_{M}\left(I \otimes_{R} T\right)=r_{(M \cap R)}(I)=1$ and $[T]-\left[I \otimes_{R} T\right] \in \mathrm{Cl}(T)$. By Proposition 5.6 there exists a finitely generated 
projective $R$-module $J$ such that $[R]-[J] \in \mathrm{Cl}(R)$ and $\left[J \otimes_{R} T\right]=\left[I \otimes_{R} T\right]$. But $J \otimes_{R} S \cong J \otimes_{R} T \otimes_{T} S$, whence $\left[J \otimes_{R} S\right]=\left[I \otimes_{R} S\right]=\left[I^{\prime}\right]$ as required.

Now let $R$ be an HNP ring and $S$ be a localization with respect to a complete deleted set of unfaithful simple left $R$-modules. We wish to compare the exact sequences

$$
0 \rightarrow K_{0}(\tau) \rightarrow K_{0}(R) \rightarrow K_{0}(S) \rightarrow 0
$$

and

$$
0 \rightarrow \mathrm{Cl}(R) \rightarrow K_{0}(R) \rightarrow H_{0}(R) \rightarrow 0 .
$$

Recall that since $S$ is a Dedekind prime ring, $K_{0}(S) \cong \mathrm{Cl}(S) \oplus Z$ by [4, 2.4]. So if $I$ is some uniform right ideal, we can rewrite the top sequence as

$$
0 \rightarrow K_{0}(\tau) \oplus Z[I] \rightarrow K_{0}(R) \rightarrow \mathrm{Cl}(S) \rightarrow 0
$$

We now proceed to show that these exact sequences are just the two exact sequences that occur from the decomposition $K_{0}(R) \cong \mathrm{Cl}(R) \oplus H_{0}(R)$. We first need some more detailed information about the states $s_{M}$.

PROPOSITION 5.9. Let $R$ be an HNP ring, let $M$ be an idempotent maximal ideal of $R$ and let $V$ be a simple right $R$-module such that $V M=0$. If $U$ is a simple $R$-module whose dual is unfaithful, then

$$
s_{M}([U]) \neq 0 \Leftrightarrow U=V \text { or } U=V_{!} .
$$

ProOF. It is clear that $s_{M}([V]) \neq 0$; hence it suffices to show that if $U \not V$, then $s_{M}([U]) \neq 0$ if and only if $U=V_{\text {! }}$. So suppose that $U \not V$ and let $I$ be a maximal right ideal such that $R / I \cong U$. In this case, the following equivalences are established fairly easily:

$$
\operatorname{Ext}_{R}^{1}(U, V)=0 \Leftrightarrow R / I M \text { is semisimple } \Leftrightarrow I M=I \cap M .
$$

On the other hand,

$$
s_{M}([U])=0 \Leftrightarrow l(I / I M)=l(R / M) \Leftrightarrow I M=I \cap M .
$$

But as observed in Theorem 3.2,U $=V_{\text {! }}$ if and only if $\operatorname{Ext}_{R}^{1}(U, V) \neq 0$.

LEMMA 5.10. $\mathrm{Cl}(R) \cap\left(K_{0}(\tau) \oplus Z[I]\right)=0$.

Proof. Let $x \in \mathrm{Cl}(R) \cap\left(K_{0}(\tau) \oplus Z[I]\right)$. Since $s_{0}([U])=0$ for all $U \in \tau$ and $s_{0}([I])=1$, it is clear that the $Z[I]$ component of $x$ must be zero. Hence $x \in K_{0}(\tau)$. Now $K_{0}(\tau)$ is a free abelian group on the isomorphism classes of simple modules in $\tau$. Let $\operatorname{supp}(x)$ be the set of simple modules which occur in the expression for $x$. Since $\operatorname{supp}(x)$ is finite and $\tau$ contains no cycles of simple modules we can find a simple module $V$ such that $[V] \in \operatorname{supp}(x)$ but $\left[V^{!}\right] \notin \operatorname{supp}(x)$. Let $M=\operatorname{ann}\left(V^{!}\right)$. Then $s_{M}([U])=0$ for all $U$ not isomorphic to $V$ in $\operatorname{supp}(x)$ by Proposition 5.9. Applying $s_{M}$ to $x$ implies that the coefficient of $[V]$ is zero, a contradiction. 
THEOREM 5.11. Let $R$ be an HNP ring. Then the following commutative diagram is exact.

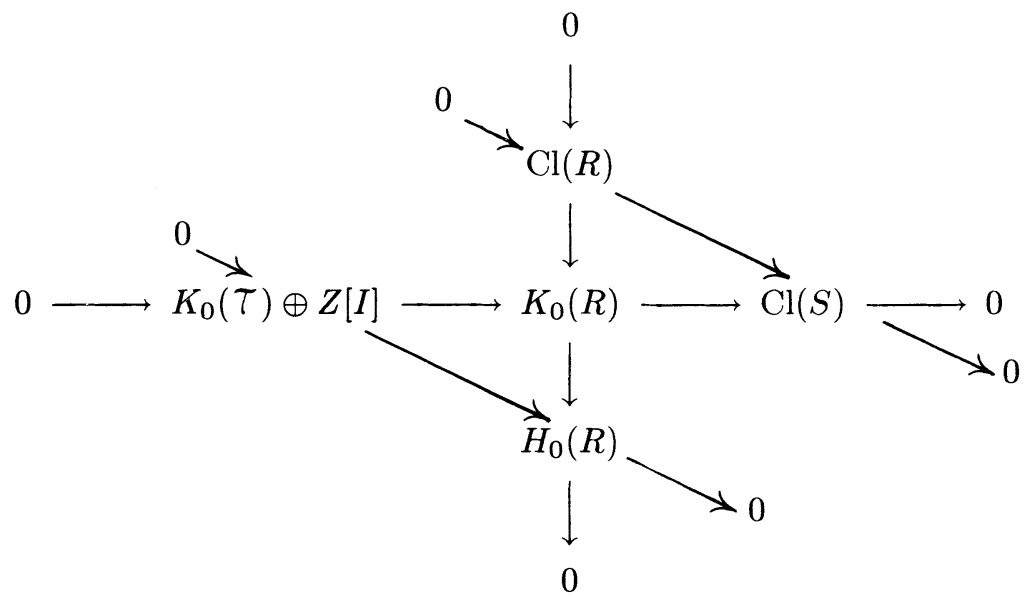

In particular $\mathrm{Cl}(R) \cong \mathrm{Cl}(S)$ and the short exact sequences both split.

ProOF. The surjectivity of the top diagonal follows from Theorem 5.8. The injectivity of the bottom diagonal follows from Lemma 5.10. Exactness then follows immediately.

COROLLARY 5.12. Let $R$ be an HNP ring with finitely many idempotent ideals. Then $K_{0}(R) \cong \mathrm{Cl}(R) \oplus Z^{t+1}$ where $t$ equals the number of idempotent maximal ideals minus the number of cycles.

Finally we mention two more corollaries to Theorem 5.11. The first is a generalization of a result of Jacobinski [9] for hereditary orders over Dedekind domains.

COROLLARY 5.13. Let $R$ be an HNP ring and let $T$ be a ring contained between $R$ and $a$ Dedekind closure of $R$. Then there is a natural isomorphism $\beta: \operatorname{Cl}(R) \rightarrow$ $\mathrm{Cl}(T)$ given by $\beta[A]=\left[A \otimes_{R} T\right]$.

Proof. Let $S$ be a Dedekind closure of $R$ containing $T$ and let $\alpha: \operatorname{Cl}(R) \rightarrow$ $\mathrm{Cl}(S)$ and $\gamma: \mathrm{Cl}(T) \rightarrow \mathrm{Cl}(S)$ be the analogously defined maps. Then $\alpha$ and $\gamma$ are both isomorphisms by Theorem 5.11. But clearly $\alpha=\gamma \beta$ so $\beta$ is also an isomorphism.

If $T_{1}$ and $T_{2}$ are Dedekind prime rings right equivalent to $R$, then $T_{1}$ and $T_{2}$ are Morita equivalent, whence obviously $K_{0}\left(T_{1}\right) \cong K_{0}\left(T_{2}\right)$. Our final result says that this isomorphism holds for any pair of Dedekind closures of an HNP ring.

COROLlaRY 5.14. Let $R$ be an HNP ring and let $T_{1}$ and $T_{2}$ be Dedekind closures of $R$. Then $K_{0}\left(T_{1}\right) \cong K_{0}\left(T_{2}\right)$.

Proof. Since $T_{1}$ and $T_{2}$ are Dedekind, $K_{0}\left(T_{i}\right) \cong \mathrm{Cl}\left(T_{i}\right) \oplus Z$ for $i=1$ and 2 . But $\mathrm{Cl}\left(T_{1}\right) \cong \mathrm{Cl}(R) \cong \mathrm{Cl}\left(T_{2}\right)$ by Corollary 5.13 .

\section{REFERENCES}

1. H. Bass, Algebraic K-theory, Benjamin, New York, 1968.

2. D. Eisenbud and J. C. Robson, Modules over Dedekind prime rings, J. Algebra 16 (1970), 67-85. 
3. __ Hereditary noetherian prime rings, J. Algebra 16 (1970), 86-104.

4. K. R. Goodearl, Localization and splitting in hereditary noetherian prime rings, Pacific J. Math. 53 (1974), 137-151.

5. _ The state space of $K_{0}$ of a ring, Ring Theory, Waterloo 1978 (D. Handelman and J. Laurence, eds.), Lecture Notes in Math., vol. 734, Springer-Verlag, Berlin and New York, 1979, pp. 91-117.

6. K. R. Goodearl and R. B. Warfield, Jr., Simple modules over hereditary Noetherian prime rings, J. Algebra 57 (1979), 82-100.

7. $\ldots$, State spaces of $K_{0}$ of Noetherian rings, J. Algebra 71 (1981), 322-378.

8. R. Gordon and J. C. Robson, Krull dimension, Mem. Amer. Math. Soc., No. 133 (1973).

9. H. Jacobinski, Two remarks about hereditary orders, Proc. Amer. Math. Soc. 28 (1971), 1-8.

10. A. V. Jategaonkar, Localisation in Noetherian rings, London Math. Soc. Lecture Note Series 89, Cambridge Univ. Press, Cambridge, 1985.

11. J. Kuzmanovich, Localisations of HNP rings, Trans. Amer. Math. Soc. 173 (1972), 137-157.

12. J. Milnor, Introductin to algebraic K-theory, Princeton Univ. Press, Princeton, N.J., 1971.

13. D. Quillen, Higher algebraic $K$-theory. I, Algebraic $K$-Theory, vol. 1 (H. Bass, ed.), Lecture Notes in Math., vol. 341, Springer-Verlag, Berlin and New York, 1973.

14. I. Reiner, Maximal orders, Academic Press, New York, 1975.

15. J. C. Robson, Idealizers and hereditary noetherian prime rings, J. Algebra 22 (1972), 45-81.

16. J. T. Stafford, Generating modules efficiently: algebraic $K$-theory for noncommutative noetherian rings, J. Algebra 69 (1981), 312-346.

17. J. T. Stafford and R. B. Warfield, Hereditary orders with infinitely many idempotent ideals, J. Pure Appl. Algebra 31 (1984), 217-226.

18. _ Constructions of hereditary Noetherian and simple rings, Proc. London Math. Soc. 51 (1985), 1-20.

19. B. Stenstrom, Rings of quotients, Springer-Verlag, New York, 1975.

20. R. B. Warfield, Jr., The number of generators of a module over a fully bounded ring, J. Algebra 66 (1980), 425-447.

21. A. Zaks, Hereditary Noetherian rings, J. Algebra 29 (1974), 513-527.

Department of Mathematical Sciences, University of Cincinnati, CincinNATI, OHIO 45221 